\title{
Contrast-induced encephalopathy after coronary angioplasty and stent implantation
}

Jun-Liang Yuan ${ }^{1}$, Shuang-Kun Wang ${ }^{2}$, Xiao-Juan Guo ${ }^{2}$, Ling-ling Ding ${ }^{1}$, Wen-Li Hu ${ }^{1}$

${ }^{1}$ Department of Neurology, Beijing Chaoyang Hospital, Capital Medical University, Beijing, China

2Department of Radiology, Beijing Chaoyang Hospital, Capital Medical University, Beijing, China

Submitted: 1 May 2016

Accepted: 15 June 2016

Arch Med Sci Atheroscler Dis 2016; 1: e63-e65

DOI: 10.5114/amsad.2016.61000

Copyright @ 2016 Termedia \& Banach

Contrast-induced encephalopathy $(\mathrm{CIE})$ is a very rare complication of coronary angiography [1]. Clinical presentations include seizures, mental alterations, cortical blindness, ophthalmoplegia and other focal neurological deficits [2,3], which make it difficult to distinguish it from other well-known complications such as subarachnoid hemorrhage (SAH) [4]. The mechanism and causes of neurotoxicity are still controversial. The neurotoxic effects of iodinated contrast media may be attributed to the disruption of a temporary blood brain barrier (BBB) [5]. The prognosis is considered to be favorable, with rapid clinical resolution. To our best knowledge, there are only rare case reports describing CIE following use of contrast agents on these complications after coronary angiography [4].

Here, we present two patients with coronary heart disease in the acute stage who developed CIE following coronary angioplasty with the non-ionic contrast agent iopromide (Ultravist R370). A 71-year-old woman (case 1) and a 50-year-old woman (case 2) received a large dose of intravascular contrast medium (about $200 \mathrm{ml}$ ) for coronary angioplasty with the iodinated contrast agent. Implantation of six stents was performed in case 1 (three in the right coronary artery, three in the left anterior descending coronary artery), and three stents were implanted in case 2 (two in the left anterior descending coronary artery, one in the left coronary circumflex artery). About $2 \mathrm{~h}$ after coronary angioplasty, the first patient only suffered from somnolence (case 1), and the second patient complained of headache, dizziness and vomiting (case 2). Neurological exanimation showed no abnormalities in the two patients. Initial computed tomography (CT) of the brain performed immediately appeared to show diffuse high densities in the subarachnoid space (Figures $1 \mathrm{~A}-\mathrm{D}$ ), and the contrast agent was reabsorbed from the subarachnoid space by the next day (Figure $1 \mathrm{E}-\mathrm{H}$ ). The second patient had very similar clinicoradiologic characteristics (Figure 2), and such abnormalities disappeared quickly on the second day. Both patients' renal status and thyroid function were normal.

The administration of contrast agents is usually associated with very few side effects [6]. Seizures, alterations in mental state, cortical blindness and ophthalmoplegia are known side effects of contrast agents [2, 7-9]. It is difficult to distinguish CIE from other well-known complications such as SAH $[7,10]$. Here, we report self-limiting encephalopathy in 2 patients following the administration of intracoronary iopromide

\author{
Corresponding author: \\ Dr. Wen-Li Hu \\ Department of Neurology \\ Beijing Chaoyang Hospital \\ Capital Medical University \\ Beijing 100020, China \\ Phone: +861085231398 \\ E-mail: huwenli@sina.com
}



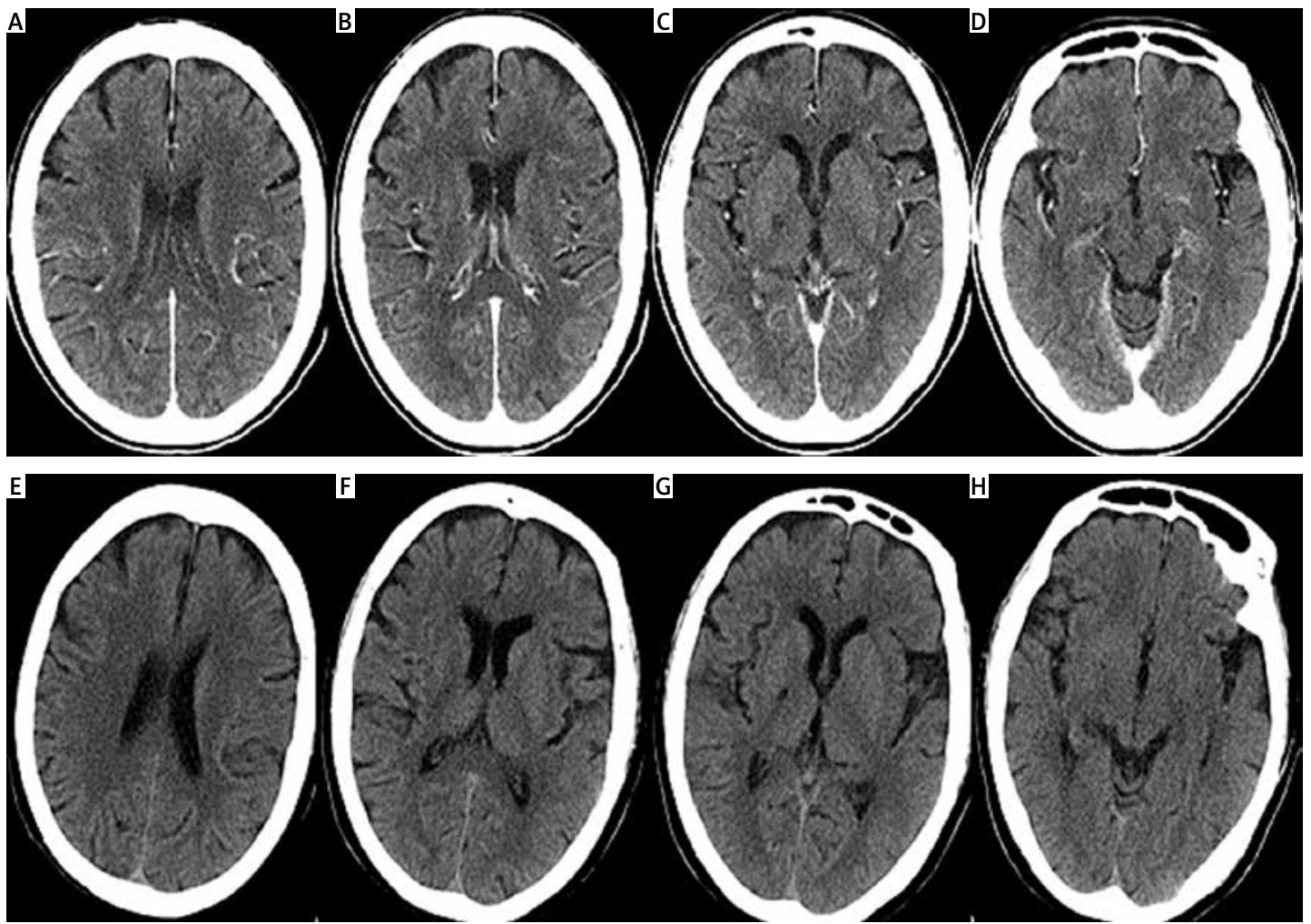

Figure 1. Head CT findings of a 71-year-old female patient after coronary angioplasty and the following day (case 1). Noncontrast CT scan at presentation (A-D) shows diffuse high densities in subarachnoid space. On the next day, repeat noncontrast $\mathrm{CT}$ scan shows marked improvement, revealing that the contrast agent was reabsorbed from the subarachnoid space $(\mathrm{E}-\mathrm{H})$, and the level of consciousness completely recovered
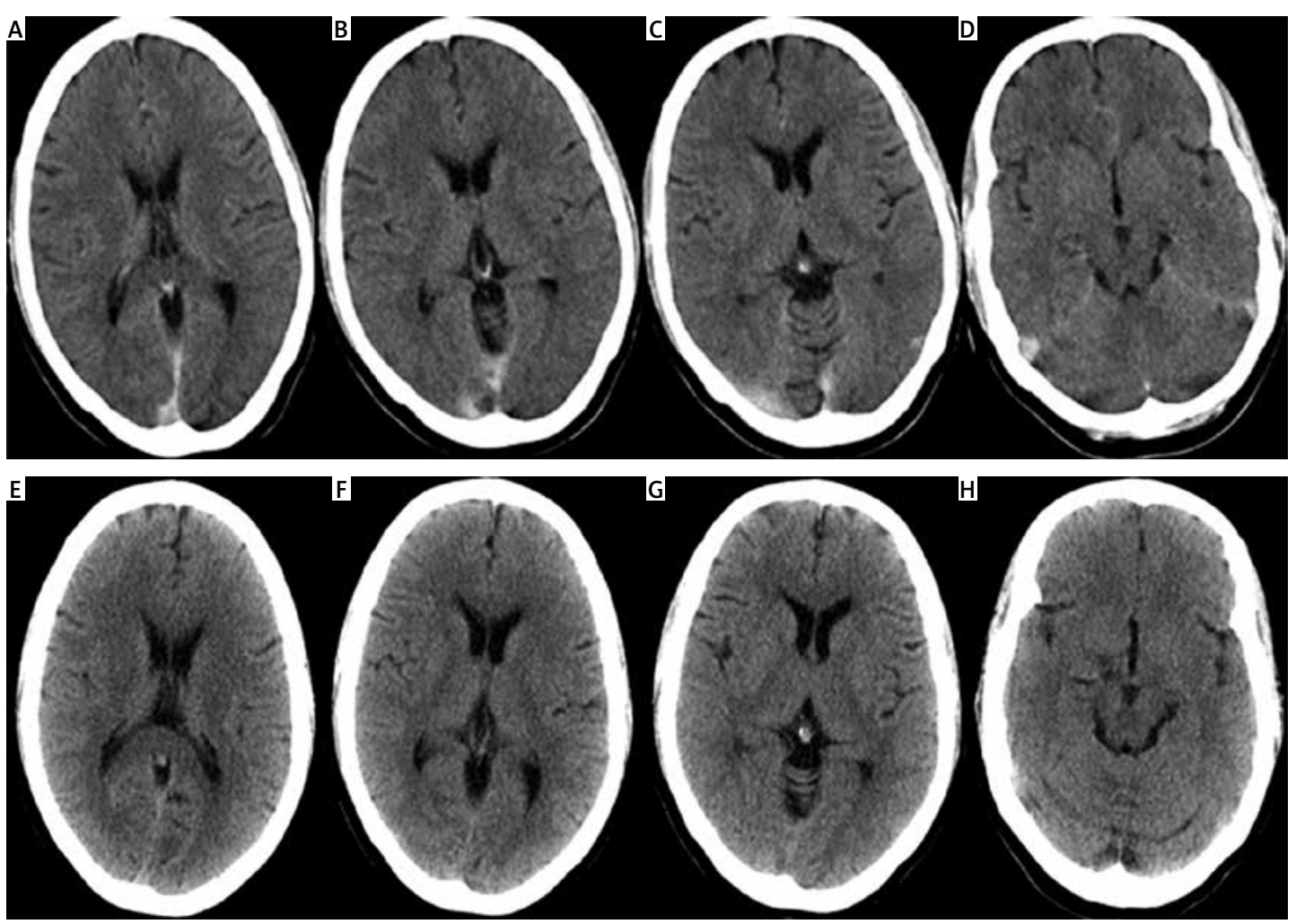

Figure 2. A 50-year-old female patient who has sulcal hyperintensity on noncontrast $\mathrm{CT}$ at presentation and the next day (case 2). Noncontrast CT scan at presentation (A-D) shows high densities in contrast agent in superior sagittal sinus and right occipital lobe. One day later, repeat noncontrast CT scan indicates resolution of such abnormalities $(\mathrm{E}-\mathrm{H})$, with neurological symptoms having improved rapidly 
after coronary angiography, which presented very likely as $\mathrm{SAH}$. The mechanism and causes of such neurotoxicity are controversial. The radiological findings were compatible with temporary disruption of and leakage through the BBB [11]. A high dose of contrast, perhaps in combination with an uncharacterized individual vulnerability, may result in acute breakdown of the BBB, allowing entrance of contrast material into the brain and the acute development of a dramatic clinical presentation [12]. Diagnosis of contrast encephalopathy is made by demonstrating typical CT findings in a symptomatic patient after exclusion of thromboembolic and hemorrhagic complications [4].

Due to the spontaneous clinicoradiologic improvement, we hypothesize that an illusion of cerebral high densities in the subarachnoid space may be caused by contrast medium extravasation [13]. Despite the benign and self-limiting nature of contrast encephalopathy [8], it may be an early warning sign of future nervous system disease, which requires careful evaluation and accurate management. Thus, immediate follow-up of these patients is necessary.

However, some limitations should be considered. Firstly, it is very regretful that we did not perform brain magnetic resonance imaging (MRI) to identify the minor changes from the subarachnoid space in these 2 cases, in spite of brain MRI not being sufficiently sensitive to identify cerebral hemorrhage, especially SAH. Secondly, if we could have applied some other imaging techniques (such as PET or SPECT) that more clearly depict leakage of contrast into the subarachnoid space, our hypothesis of dysfunction of the BBB might be much more convincing.

In conclusion, although very rare, CIE may occur during coronary intervention without a particular predisposition. Despite it being a benign and self-limiting encephalopathy, it also can potentially cause permanent neurological deficits or death [2]. As a result, it may be an early warning sign of future nervous system disease during the procedure of coronary angioplasty for vascular interventional physicians in clinical trials.

\section{Acknowledgments}

This work was supported by the National Natural Science Foundation of China (81301016).

\section{Conflict of interest}

The authors declare no conflict of interest.

\section{References}

1. Guimaraens L, Vivas E, Fonnegra A, et al. Transient encephalopathy from angiographic contrast: a rare com- plication in neurointerventional procedures. Cardiovasc Intervent Radiol 2010; 33: 383-8.

2. Kocabay G, Karabay CY, Kalayci A, et al. Contrast-induced neurotoxicity after coronary angiography. Herz 2014; 39: 522-7.

3. Yu J, Dangas G. Commentary: new insights into the risk factors of contrast-induced encephalopathy. J Endovasc Ther 2011; 18: 545-6.

4. Leong S, Fanning NF. Persistent neurological deficit from iodinated contrast encephalopathy following intracranial aneurysm coiling. A case report and review of the literature. Interv Neuroradiol 2012; 18: 33-41.

5. Iwata T, Mori T, Tajiri H. Repeated injection of contrast medium inducing dysfunction of the blood-brain barrier: case report. Neurol Med Chir (Tokyo) 2013; 53: 34-6.

6. Singh J, Daftary A. lodinated contrast media and their adverse reactions. J Nucl Med Technol 2008; 36: 69-74.

7. Sharp S, Stone J, Beach R. Contrast agent neurotoxicity presenting as subarachnoid hemorrhage. Neurology 1999; 52: 1503-5.

8. Sawaya RA, Hammoud R, Arnaout S, et al. Contrast-induced encephalopathy following coronary angioplasty with iohexol. South Med J 2007; 100: 1054-5.

9. Kocabay G, Karabay CY. Iopromide-induced encephalopathy following coronary angioplasty. Perfusion 2011; 26: 67-70.

10. Potsi S, Chourmouzi D, Moumtzouoglou A. Transient contrast encephalopathy after carotid angiography mimicking diffuse subarachnoid haemorrhage. Neurol Sci 2012; 33: 445-8.

11. Junck L, Marshall WH. Neurotoxicity of radiological contrast agents. Ann Neurol 1983; 13: 469-84.

12. Torvik A, Walday P. Neurotoxicity of water-soluble contrast media. Acta Radiol Suppl 1995; 399: 221-9.

13. Foltys $H$, Krings $T$, Block F. Cerebral contrast medium extravasation after coronary angioplasty. Nervenarzt 2003; 74: 892-5. 